Research Article

\title{
Influence Diagnostics in Log-Normal Regression Model with Censored Data
}

\author{
Javeria Khaleeq $\mathbb{D}^{\mathrm{D}},{ }^{1}$ Muhammad Amanullah, ${ }^{1}$ and Zahra Almaspoor ${ }^{2}{ }^{2}$ \\ ${ }^{1}$ Department of Statistics, Bahauddin Zakariya University, Multan 60800, Pakistan \\ ${ }^{2}$ Department of Statistics, Yazd University, P.O. Box 89175-741, Yazd, Iran \\ Correspondence should be addressed to Zahra Almaspoor; z.almaspoor@stu.yazd.ac.ir
}

Received 3 October 2021; Accepted 24 November 2021; Published 20 December 2021

Academic Editor: Dost Muhammad Khan

Copyright (C) 2021 Javeria Khaleeq et al. This is an open access article distributed under the Creative Commons Attribution License, which permits unrestricted use, distribution, and reproduction in any medium, provided the original work is properly cited.

\begin{abstract}
Dealing with the biological data, the skewed distribution is approximated by the Log-Normal Regression model (LNRM). Traditional estimation techniques for the LNRM are sensitive to unusual observations. These observations greatly affect the model analysis, which makes imprecise conclusions. To overcome this issue, we proposed to develop diagnostics measures based on local influence diagnostics to identify such curious observations in the LNRM under censoring. The proposed measures are derived by perturbing the case weight, response, and explanatory variables. Furthermore, we also consider the One-Step Newton-Raphson method and generalized cook's distance. We study the Monte Carlo simulation and its application to real data to illustrate the developed approaches.
\end{abstract}

\section{Introduction}

Survival analysis has seen a great surge of research interests over the last three decades $[1,2]$. It is usually used to analyze an event of interest that occurs within a specified period of time. The accelerated failure time (AFT) model is a very common method because it directly expresses the failure time rather than the probability as in the proportional hazard model and therefore would be an important alternative to the proportional hazard model $[3,4]$. The AFT model makes modeling simple as it takes the logarithm of the failure time linearly to the covariates $[5,6]$.

The log-normal distribution (LND) has a wide application in biology [7], hydrology [8], and social sciences [9]. It has also been used to fit a different kind of cancer survival data [10]. It is also evident from articles [11-14] and the references cited therein. Very recently, the LND has also been applied in COVID-19 [15] and financial decisions [16]. Sweet [17] considered the hazard rate in the LND.

The detection of unusual observations is difficult, and the impacts of their presence on various aspects of the linear statistical model are very well-known [18]. When there are influential observations in a data set, various diagnostic methods have been suggested, which are common in practice. Among these methods, case omission has got special attention. Cook [19] has introduced the case omission, and a large number of articles on the subject have been published since then. Zhu et al. [20-22] provided extended versions of case omission for several statistical models. Recently, the case omission approach has been extended to a censored log-linear regression model. One may use Student- $t$ censored regression model in the different versions of the case omission technique [23]. Apart from this paper, no attention has been given to the impacts of influential observations on various aspects of the estimates of LNRM.

Cook [24] provided an approach known as curvature diagnostics. Escobar and Meeker [25] described the implication method of local influence methods for the detection of data/model perturbations to get significant effects on maximum likelihood estimates based on censoring. Weissfeld and Schneider [26, 27] discussed local influence (LI) trends for Weibull models and normal linear models. Leiva et al. [28] developed LI methods for the generalized linear model (GLM) having the error distribution as log- 
Birnbaum-Saunders distribution, and Ortega et al. [29] investigated the exponentiated Weibull distributed model. For censoring, Liu [30] investigated LI in elliptical linear models, Ortega et al. [31] adopted the LI technique for the GLM, and Venezuela et al. [32] discussed the diagnostics technique for the GLM based on LI. Lachos et al. [33] used the LI approach in Grubb's model, Paula et al. [34] investigated the LI technique in linear models with the first-order autoregressive elliptical error. Russo et al. [35] employed the LI technique in nonlinear-mixed-effects elliptical models. Vanegas et al. [36] examined the performance of LI for linear regression models in Weibull distribution. Very recently, Khaleeq et al. [37] used influence diagnostic techniques in the case of censoring by using a log-logistic regression model.

In this paper, curvature diagnostics were developed for the LNRM based on LI and case omission approach when censoring is present in the data. Diagnostic performance for the comparison of the developed approaches with the available measures is also presented.

The structure of this paper is as follows: Section 2 is based on the LND. Section 3 presents the formulation of the LNRM under censoring. The diagnostic techniques with derived approaches are in Section 4. Section 5 employs Monte Carlo simulation and real-world examples for the empirical performance of the derived techniques. The last section presents the findings and conclusion.

\section{Log-Normal Distribution}

The LND is also known as the Galton or Galton's distribution, named after Francis Galton (1879), a statistician during the English Victorian Era. The focus of interest is the occurrence of a particular event of interest within a given period of time. The response of variable $T$ is a nonnegative random variable which gives the survival time of an object or an individual, which can be expressed as a probability density function denoted by $f(t ; \lambda, \gamma)$ with parameters $\lambda$ and $\gamma$,

$$
f(t ; \lambda, \gamma)=(2 \pi)^{-1} t^{-1} \exp \left[\frac{-\gamma^{2}(\log \lambda t)^{2}}{2}\right],
$$

where $\gamma>0$ is the scale parameter and $\lambda>0$ is the shape parameter. Survival function corresponding to the random variable $T$ with the LND density is given by

$$
S(t ; \lambda, \gamma)=P(T \geq t)=1-(\gamma \log \lambda t) .
$$

This family of distribution is suitable when the hazard rate initially increases and then decreases at times, can be hump-shaped, and has a nonmonotonic failure rate.

\section{The LNRM for Censored Data}

Let $\mathbf{x}=\left(x_{1}, x_{2}, \ldots, x_{p}\right)^{T}$ be covariate vector associated by a regression model to responses $y=\log (T)$. Considering the transformation $\gamma=1 / \sigma$ and $\lambda=\exp (-\mu)$, it follows that the density function of $Y$ can be written as

$$
f(y)=\frac{1}{\sigma} \frac{1}{\sqrt{2 \pi}} \frac{\exp -((y-u) / \sigma)^{2}}{2}
$$

where $y>0$ and $-\infty<u<\infty$ Using (3), AFT model is given as

$$
Y=\mu+\sigma w
$$

where $u=x^{T} \beta$ and variable $w$ follows the density

$$
f(w)=\frac{1}{\sqrt{2 \pi}} \frac{\exp -(w)^{2}}{2}, \quad \forall-\infty<w<\infty,
$$

with survival function given by

$$
S(w)=1-\left(\frac{(y-\mu)}{\sigma}\right) .
$$

Now consider the regression model by using the LND in (4). Presenting responses $y$ and $x$ as the covariate vectors, $y \mid x$ can be represented as

$$
y_{i}=x_{i}^{T} \beta+\sigma w_{i}, \quad i=1,2, \ldots, n,
$$

where $\beta=\left(\beta_{1}, \ldots, \beta_{p}\right)^{T}, \sigma>0, x_{i}^{T}=\left(x_{1 i}, x_{2 i}, \ldots, x_{p i}\right)$ is the vector of explanatory variables, and $w_{i}$ follows the distribution in (6).

Moreover, corresponding to the sample $\left(y_{1}, x_{1}\right),\left(y_{2}, x_{2}\right), \ldots,\left(y_{n}, x_{n}\right)$ from (3), $y_{i}$ symbolizes the logarithm of $T_{i}$ and $x_{i}$ symbolizes the covariate vector of $i^{\text {th }}$ individual. We can explain the log-likelihood function as

$$
L(\beta, \sigma)=\sum_{i=1}^{n}\left[-\delta_{i} \ln \sigma-\delta_{i} \ln \sqrt{2 \pi}-\delta_{i} \frac{w_{i}^{2}}{2}-\ln \left(w_{i}\right)+\delta_{i} \ln \left(w_{i}\right)\right],
$$

where $w_{i}=\left(y_{i}-x_{i}^{T} \beta\right) / \sigma$. The maximum likelihood estimates (MLE) for the parameter vector $\theta=\left(\beta^{T}, \sigma\right)^{T}$ can be attained by Newton-Raphson (NR) approach. Covariance for MLEs $\hat{\theta}$ can also be obtained by using the Hessian matrix. The asymptotic covariance matrix is given by $(I(\widehat{\theta}))^{-1}$ with $I(\widehat{\theta})=-E[\ddot{L}(\widehat{\theta})]$ such that $\ddot{L}(\widehat{\theta})=\left\{\partial^{2} l(\widehat{\theta}) / \partial \theta \partial \theta^{T}\right\}$.

Due to the fact that the mechanism of censoring determination of Fisher information matrix $I(\widehat{\theta})$ is not possible, then in its place, the matrix of the second derivatives of the log-likelihood $(-\ddot{L}(\hat{\theta}))$ is used, which is given as

$$
\ddot{L}(\widehat{\theta})=\left(\begin{array}{cc}
L_{\beta \beta} & L_{\beta \sigma} \\
\cdot & L_{\sigma \sigma}
\end{array}\right),
$$

with the submatrices in Appendix.

\section{Diagnostic Analysis}

Influence diagnostic methods evaluate the sensitivity of the parameter estimation particular model under the perturbation either in the data set or in the essential assumptions for the model. In this section, a few popular approaches to diagnostics are discussed.

4.1. Global Influence Diagnostics. The alternative name of the case omission approach is global influence diagnostics; a popular technique is an estimation process to assess the 
removing effect of an observation from the data set. The common methods are given as follows.

4.1.1. Generalized Cook Distance. The coefficient estimation has major importance in regression modeling. Cook distance [24] defines the effect of omitting cases on estimated coefficients $[24,38]$. The cook distance $\left(\mathrm{CD}_{i}\right)$ is the standardized norm of $\left(\widehat{\theta}_{(i)}-\widehat{\theta}\right)$ for LNRM depending on vector $\theta=\left[\beta^{T}, \sigma\right]^{T}$ which describe recognize the global influence, i.e., the generalized $\mathrm{CD}_{i}\left(\mathrm{GCD}_{i}\right)$, defined as,

$$
\operatorname{GCD}_{i}(\widehat{\theta})=\frac{1}{p+1}\left[\left(\widehat{\theta}_{(i)}-\widehat{\theta}\right)^{T}[\ddot{L}(\widehat{\theta})]^{-1}\left(\widehat{\theta}_{(i)}-\widehat{\theta}\right)\right],
$$

where $i=1, \ldots, n$ and $p$ shows the number of coefficients of regression.

Remark 1. Larger value for $\mathrm{GCD}_{i}(\widehat{\theta})$ states the significant influence of $i^{\text {th }}$ observation on MLEs of $\theta$. Zhu et al. [20] presented that the average of $\operatorname{GCD}_{i}(\hat{\theta})$ is almost $1 / n$, such that $2 / n$ can be used as a cut-off point for $\operatorname{GCD}_{i}(\widehat{\theta})$, $i=1,2, \ldots, n$.

4.1.2. One-Step Newton-Raphson Method. Single observation influence can be ascertained by the deletion approach on estimation. In this approach, $i^{\text {th }}$ observation is removed from data, and estimation of the parameter is calculated $[38,39]$. In LNRM estimation, consider iteration and onestep approximation; that is, the NR approach to the eliminated value of $\theta=(\beta, \sigma)$ is

$$
\Delta \mathrm{NR}=\widehat{\theta}-\widehat{\theta}_{(i)}=I_{(i)}^{-1}(\widehat{\theta}) q_{i}(\widehat{\theta}),
$$

where $q_{i}$ is the $i^{\text {th }}$ element of $q(\hat{\theta})$ and $I_{(i)}$ with $i^{\text {th }}$ point eliminated presented in Appendix.

4.2. Local Influence. The LI approach is based on geometric differentiation in spite of case deletion. A differential comparison of estimation is required before and after perturbing the model and data. Different schemes exist to determine the LI.

4.2.1. The Local Influence Diagnostics. For LI diagnostics, $l(\widehat{\theta})$ is postulated $\log$-likelihood with vector $\theta=\left(\beta^{T}, \sigma\right)^{T}$ and vector $u_{n \times 1}$ is a vector of perturbation to some open subset $\Omega \subset \mathbb{R}^{n}$. The log-likelihood function under the perturbation of case weight is

$$
l\left(\frac{\widehat{\theta}}{u}\right)=\sum_{i \in F} u_{i} \log f\left(y_{i} ; \theta\right)+\sum_{i \in C} u_{i} \log S\left(y_{i} ; \theta\right),
$$

where $0 \leq u_{i} \leq 1$ and $u_{0}=(1,1, \ldots, 1)^{T}$ is the no perturbation vector. Note that $l\left(\widehat{\theta} / u_{0}\right)=l(\widehat{\theta})$. Cook [24] weighed the outcome of a definite perturbation by likelihood displacement by taking the performance of the surface. We are concerned with the elimination of conceivably unusual (influential) observations. The likelihood displacement, that is, $\operatorname{LD}(\widehat{u})=2\left[l(\widehat{\theta})-l\left(\widehat{\theta}_{u}\right)\right]$, measures the distance between $\widehat{\theta}$ and $\widehat{\theta}_{u}$, where $\widehat{\theta}_{u}$ is the vector of perturbed MLE. The normal curvature for $\theta$ at the direction $\|d\|=1$ is given by $2\left|d^{T} \Delta^{T}(\ddot{L})^{-1} \Delta d\right|$, where $-\ddot{L}$ is attended hypothesize Fisher information matrix with $\left(u=u_{0}\right)$ and $\Delta$ is $(p \times 1) \times n$ ordered matrix with elements

$$
\Delta_{i j}=\frac{\partial^{2} L(\widehat{\theta} / u)}{\partial \theta_{i} \partial u_{i}}
$$

evaluated at $\theta=\widehat{\theta}$ and $u=u_{0}$, where $\hat{\theta}$ and $u_{0}$ are the perturbed vectors [24]. The $\mathrm{CV}_{\max }$ of the matrix $B=\Delta^{T}(\ddot{L})^{-1} \Delta$ has the largest eigenvalue. Point out the unusual cases with the cut-off point for LI as

$$
\begin{aligned}
C_{i} & \geq 2 \bar{C}, \\
\bar{C} & =\frac{1}{n} \sum C_{i} .
\end{aligned}
$$

4.2.2. Case-Weights Perturbation. The log-likelihood function of the LNRM with $\mathbf{u}=\left(u_{1}, u_{2}, \ldots, u_{n}\right)^{T}$ (vector of weights) takes the form

$$
l\left(\frac{\widehat{\theta}}{u}\right)=\sum_{i=1}^{n} u_{i}\left[-\delta_{i} \ln \sigma-\delta_{i} \ln \sqrt{2 \pi}-\delta_{i} \frac{w_{i}^{2}}{2}-\ln \left(w_{i}\right)+\delta_{i} \ln \left(w_{i}\right)\right],
$$

where $0 \leq u_{i} \leq 1$ and $u_{0}=(1,1, \ldots, 1)^{T}$. We find, after some algebraic manipulation, the following expressions for the weighted log-likelihood function and the elements of the matrix $\Delta$.

$$
\Delta_{i j}=\left\{\begin{array}{c}
\frac{x_{i j}}{\sigma}\left(\delta_{i} w_{i}+\frac{1}{w_{i}}-\frac{\delta_{i}}{w_{i}}\right) \\
\frac{1}{\sigma}\left(1+\delta_{i} w_{i}^{2}-2 \delta_{i}\right)
\end{array}\right\} .
$$

4.2.3. Response Perturbation. Consider the regression model (7) by assuming now that each $y_{i}$ is perturbed as $y_{i} \longrightarrow y_{i}+u_{i} S_{y}=y_{i}^{*}, i=1,2, \ldots, n$, where $S_{y}$ is a scale parameter. For the perturbation, in response to the LNRM, the log-likelihood takes the form

$$
l\left(\frac{\widehat{\theta}}{u}\right)=\sum_{i=1}^{n}\left[-\delta_{i} \ln \sigma-\delta_{i} \ln \sqrt{2 \pi}-\delta_{i} \frac{\left(w_{i}^{*}\right)^{2}}{2}-\ln \left(w_{i}^{*}\right)+\delta_{i} \ln \left(w_{i}^{*}\right)\right],
$$

where $w_{i}^{*}=\left(\left(\left(y_{i}+u_{i} S_{y}\right)-x_{i}^{T} \beta\right) / \sigma\right), i=1,2, \ldots, n$. When the response is perturbed, the log-likelihood function with manipulated expressions for matrix $\Delta$ is as follows:

$$
\Delta_{i j}=\left[\begin{array}{c}
\frac{S_{y} X_{i j}}{\sigma^{2}}\left(\delta_{i}-\frac{1}{\left(w_{i}^{*}\right)^{2}}+\frac{\delta_{i}}{\left(w_{i}^{*}\right)^{2}}\right) \\
\frac{2}{\sigma^{2}}\left(\delta_{i} W_{i} S_{y}\right)
\end{array}\right] .
$$


Vector $d_{\max }\left(w_{i}\right)$ is created on $w_{i}=x_{i}$, which corresponding to the $n \times 1$ vector

$$
\begin{aligned}
& d_{\max }\left(x_{i}\right) \propto-\Delta^{T} \ddot{L}_{\beta \beta}^{-1} x_{i}, \\
& \mathrm{CV}_{\text {response (max) }}\left(y_{i}\right)=2\left|d_{\max }^{T} \ddot{L}^{-1} d_{\max }\right| .
\end{aligned}
$$

A large value for the $i^{\text {th }}$ component of the above expression, $d_{\max }\left(x_{i}\right)$, indicates that the $i^{\text {th }}$ observation requires a considerable LI on $\hat{y}_{i}$. The index plot of vector $\left(d_{\max }\left(x_{1}\right), \ldots,\left(d_{\max }\left(x_{n}\right)\right)\right)^{T}$ identifies the high influence on fitted values.

4.2.4. Covariate Perturbation. Consider now an additive perturbation on a particular continuous covariate, namely, $X_{t}$ by making $x_{i t}+u_{i} S_{t}$, where $S_{t}$ is a scaled factor and $u \in \mathbf{R}$. This perturbation scheme leads to the following expressions for the log-likelihood function:

$$
l\left(\frac{\theta}{u}\right)=\sum_{i=1}^{n}\left[-\delta_{i} \ln \sigma-\delta_{i} \ln \sqrt{2 \pi}-\delta_{i} \frac{\left(w_{i}^{*}\right)^{2}}{2}-\ln \left(w_{i}^{*}\right)+\delta_{i} \ln \left(w_{i}^{*}\right)\right],
$$

where $\quad w_{i}^{*}=\left(\left(y_{i}-x_{i}^{* T} \beta\right) / \sigma\right), i=1,2, \ldots, n$, where $x_{i}^{* T} \beta=\beta_{1}+\beta_{2} x_{2 i}+\cdots+\beta_{t}\left(x_{t i}+u_{i} S_{t}\right)+\cdots+\beta_{p} x_{p i}$. The covariate perturbation is manipulated elements of the matrix $\Delta$ is

$$
\Delta_{i j}=\left[\begin{array}{c}
\frac{X_{i j}}{\sigma^{2}}\left(\delta_{i} S_{y} \beta+\frac{S_{t} \beta}{\left(w_{i}^{*}\right)^{2}}+\frac{\delta_{i} S_{t \beta}}{\left(w_{i}^{*}\right)^{2}}\right) \\
\frac{1}{\sigma^{2}}\left(\delta_{i} w_{i}^{*} S_{y} \beta\right)
\end{array}\right] .
$$

The assessment of the larger curvature at $w=x_{i}$ leads to

$$
\mathrm{CV}_{\text {covariate (max) }}\left(x_{i}\right)=2\left|d_{\max }^{T} \ddot{L}^{-1} d_{\max }\right|,
$$

and consequently,

$$
d_{\max }\left(x_{i}\right) \propto-\Delta^{T} \ddot{L}_{\beta \beta}^{-1} x_{i} .
$$

To see to which observed values of $X_{t}$ the prediction is most sensitive under small changes in $X_{t}$, we can perform the plot of $\mathrm{CV}_{\max }\left(x_{i}\right)$ against $x_{i t}$. The index plot of the $n \times 1$ vector $\left(d_{\max }\left(x_{1}\right), \ldots, d_{\max }\left(x_{n}\right)\right)^{T}$ can indicate those observations for which a small perturbation in the value of $X_{t}$ leads to a substantial change in the prediction.

\section{Empirical Evaluation}

For the performance of the derived results, we provide a Monte Carlo simulation scheme and illustrative example with results and discussions by showing the results in tables and figures.

5.1. Simulation Study. We provide the Monte Carlo simulation scheme for the performance of developed diagnostics for LND by following a similar simulation scheme used by Ortega et al. [29] in the given section. The response variable is generated by $y_{i} \sim L N\left(\mu_{i}, \gamma\right)$, where $\mu_{i}=x_{i} \beta$ and the arbitrary values are $\gamma=1,3,9$; we select arbitrary values of true $\beta$ vector in such a manner that $\beta=1$, and the explanatory variables with no influential observations are generated by $x_{i j} \sim U(0,1)$, where $j=1,2, \ldots, p$ and $i=1,2, \ldots, n$. $n$ is set to be $50,100,150$, and 200 , and the number of explanatory variables $p$ is 1,2 , and 4 . Then, we make an influential observation in $X$ 's; that is, the 20th observation is replaced in the complete data set as $X_{20 j}=X_{20 j}+S_{y}$, $j=1,2, \ldots, p$, where $S_{y}$ is the standard deviation of response.

For this study, right random censoring is used considering the censoring observations for each of the samples generated in which $Y_{i}=\min \left(T_{i}, C_{i}\right)$ as the minimum of the survival time and the censored time of the observed time $T_{i}$ where

$$
T_{i}=\left\{\begin{array}{ll}
\delta_{i}=1, & \text { if } X \leq C \\
\delta_{i}=0, & \text { if } X>C
\end{array}\right\} .
$$

The censoring level is set to be $0 \%, 10 \%, 20 \%$, and $30 \%$. Now the performance of these diagnostics for the identification of generated influential observation with various censoring levels and with different values of dispersions is performed on the basis of the generated samples. These simulation results are performed on $R$ software.

Tables $1-3$ show the percentage results of the diagnostics measures for different explanatory variables; that is, $p=1,2$, and 4 from the simulation study. The results showed that, by increasing the levels of censoring $(0 \%, 10 \%, 20 \%$, and $30 \%)$ and $\sigma=1,3,9$, the diagnostic percentage shows a decreased trend, and by increasing the sample size $n=50,100,150,200$, a great increasing trend could be seen. By the simulation study for LNRM, the developed approaches (i.e., $\mathrm{CV}_{\text {case weight }}, \mathrm{CV}_{\text {response }}, \mathrm{CV}_{\text {covariate }}$ ) diagnosed unusual observation better in the comparison of $\mathrm{GCD}_{i}$ and One-Step NR. In the developed approaches, the performance of $\mathrm{CV}_{\text {rersponse }}$ was better in all cases at $0 \%$ censored observations. By increasing the level of censoring (10\%, 20\%, and $30 \%), \mathrm{CV}_{\text {covariate }}$ performs superior in terms of diagnosing the unusual observation in the highest percentage. Figures 1-3 display the diagnostics percentages with different level of censoring with different value of $\sigma$ at $p=1,2$, and 4 . These figures show the performance of diagnostics measures graphically, as already discussed in Tables 1-3.

5.2. Example: Ovarian Cancer Survival Data. A sample from a clinical trial of 26 ovarian cancer patient's survival time was taken from cancer treatment report [40], Mayo Clinic, Rochester, USA, to assess the effectiveness of various chemotherapies for women with ovarian cancer who had minimal residual disease after having undergone surgery to excise all tumors greater than $2 \mathrm{~cm}$ in diameter. For this study, noninformative censoring is used. The LNRM has four explanatory variables $x_{1 i}, x_{2 i}, x_{3 i}, x_{4 i}$. The first two are Age and Residual disease present in which $1=$ no, $2=$ yes. The other two are ECOG performance with dependent variable $y_{i}$ survival or censoring time and $\delta_{i}$ censoring status in which $1=$ lifetime observed, $0=$ censoring, respectively. ANR iteration is used for estimation [6]. 
TABle 1: Percentage of influential diagnostics at $p=1$.

\begin{tabular}{|c|c|c|c|c|c|c|c|}
\hline Censoring (\%) & $\sigma$ & $n$ & GCD & $\mathrm{NR}_{x_{1}}$ & $\mathrm{CV}_{\text {case weight }}$ & $\mathrm{CV}_{\text {response }}$ & $\mathrm{CV}_{\text {covariate }}$ \\
\hline \multirow{12}{*}{0} & \multirow{4}{*}{1} & 50 & 37.9 & 99.2 & 100.0 & 100.0 & 100.0 \\
\hline & & 100 & 40.5 & 95.2 & 100.0 & 100.0 & 99.6 \\
\hline & & 150 & 81.3 & 88.8 & 100.0 & 100.0 & 98.7 \\
\hline & & 200 & 82.4 & 81.6 & 100.0 & 100.0 & 98.3 \\
\hline & \multirow{4}{*}{3} & 50 & 56.7 & 99.7 & 99.6 & 100.0 & 99.9 \\
\hline & & 100 & 46.5 & 97.7 & 99.8 & 100.0 & 99.7 \\
\hline & & 150 & 73.3 & 93.6 & 100.0 & 100.0 & 99.9 \\
\hline & & 200 & 85.6 & 91.7 & 99.9 & 100.0 & 99.7 \\
\hline & \multirow{4}{*}{9} & 50 & 49.5 & 99.9 & 100.0 & 100.0 & 100.0 \\
\hline & & 100 & 61.4 & 98.5 & 99.9 & 100.0 & 99.8 \\
\hline & & 150 & 84.3 & 95.2 & 100.0 & 100.0 & 99.8 \\
\hline & & 200 & 97.2 & 91.4 & 99.6 & 100.0 & 99.9 \\
\hline \multirow{12}{*}{10} & \multirow{4}{*}{1} & 50 & 64.8 & 99.2 & 81.4 & 88.6 & 98.6 \\
\hline & & 100 & 75.9 & 93.7 & 93.8 & 83.4 & 94.2 \\
\hline & & 150 & 76.2 & 87.0 & 94.6 & 81.5 & 89.5 \\
\hline & & 200 & 77.3 & 80.4 & 95.3 & 78.8 & 81.3 \\
\hline & \multirow{4}{*}{3} & 50 & 40.4 & 98.7 & 35.2 & 87.4 & 98.6 \\
\hline & & 100 & 72.0 & 96.8 & 62.9 & 83.6 & 97.7 \\
\hline & & 150 & 70.6 & 92.0 & 73.6 & 80.9 & 95.3 \\
\hline & & 200 & 78.4 & 89.2 & 83.5 & 82.3 & 93.8 \\
\hline & \multirow{4}{*}{9} & 50 & 29.4 & 99.4 & 16.0 & 87.2 & 99.4 \\
\hline & & 100 & 59.4 & 96.3 & 33.7 & 82.3 & 98.7 \\
\hline & & 150 & 61.5 & 93.4 & 41.3 & 80.3 & 97.9 \\
\hline & & 200 & 64.7 & 90.7 & 40.5 & 78.7 & 97.1 \\
\hline \multirow{12}{*}{20} & \multirow{4}{*}{1} & 50 & 64.8 & 98.1 & 81.9 & 87.6 & 98.0 \\
\hline & & 100 & 60.3 & 94.9 & 92.1 & 79.3 & 96.0 \\
\hline & & 150 & 62.5 & 90.0 & 95.9 & 82.4 & 90.8 \\
\hline & & 200 & 57.9 & 85.1 & 98.0 & 76.6 & 86.1 \\
\hline & \multirow{4}{*}{3} & 50 & 47.4 & 98.9 & 48.5 & 85.5 & 99.5 \\
\hline & & 100 & 55.8 & 97.1 & 58.3 & 81.5 & 98.0 \\
\hline & & 150 & 61.2 & 93.1 & 71.6 & 79.1 & 95.7 \\
\hline & & 200 & 60.1 & 92.8 & 83.8 & 78.3 & 96.2 \\
\hline & \multirow{4}{*}{9} & 50 & 40.8 & 98.8 & 37.2 & 85.3 & 99.4 \\
\hline & & 100 & 50.9 & 97.0 & 40.2 & 78.4 & 99.2 \\
\hline & & 150 & 51.3 & 95.1 & 43.5 & 76.6 & 98.0 \\
\hline & & 200 & 56.7 & 93.9 & 54.3 & 78.5 & 97.6 \\
\hline \multirow{12}{*}{30} & \multirow{4}{*}{1} & 50 & 51.1 & 98.1 & 76.9 & 84.3 & 98.2 \\
\hline & & 100 & 55.4 & 95.7 & 91.0 & 82.5 & 96.6 \\
\hline & & 150 & 49.9 & 93.7 & 98.7 & 80.7 & 93.1 \\
\hline & & 200 & 45.9 & 89.1 & 99.4 & 78.3 & 86.8 \\
\hline & \multirow{4}{*}{3} & 50 & 46.9 & 98.8 & 45.5 & 85.9 & 99.2 \\
\hline & & 100 & 46.5 & 97.6 & 53.3 & 83.6 & 98.4 \\
\hline & & 150 & 48.7 & 96.3 & 79.9 & 76.7 & 97.4 \\
\hline & & 200 & 46.3 & 91.6 & 95.0 & 74.2 & 95.5 \\
\hline & \multirow{4}{*}{9} & 50 & 40.5 & 99.0 & 28.4 & 82.2 & 99.8 \\
\hline & & 100 & 45.3 & 97.9 & 43.2 & 79.1 & 99.5 \\
\hline & & 150 & 42.8 & 97.2 & 56.4 & 79.2 & 98.9 \\
\hline & & 200 & 45.9 & 96.0 & 73.0 & 70.5 & 98.0 \\
\hline
\end{tabular}

The proposed model is as follows:

$$
y_{i}=\beta_{0}+\beta_{1} x_{1 i}+\beta_{2} x_{2 i}+\beta_{3} x_{3 i}+\beta_{4} x_{4 i}+\sigma w_{i},
$$

where $w_{i}$ follows the model given in (5). The MLEs for the model parameters are estimated in the Survreg Survival package (https://rdrr.io/cran/survival/man/survreg.html) in the $\mathrm{R}$ language. The MLEs and the absolute change with respect to the unusual observations are given in Table 4 .
In Table 4, regression estimates for the full and after omitting the 1st, 13th, 14th, 21st, 22nd, 24th, and 25th observations, which was noted as influential observations and absolute changes in the estimates, were also noticed. Eliminating the 1st observation from data, absolute change results in $\beta_{4}$ as $598.6296 \%$, which displays a high influence effect for omitting observation (i.e., 1st observation). After omitting the 13 th observation, $436.4282 \%$ is the absolute 
Table 2: Percentage of influential diagnostics at $p=2$.

\begin{tabular}{|c|c|c|c|c|c|c|c|}
\hline Censoring (\%) & $\sigma$ & $n$ & GCD & $\mathrm{NR}_{x_{2}}$ & $\mathrm{CV}_{\text {case weight }}$ & $C V_{\text {response }}$ & $\mathrm{CV}_{\text {covariate }}$ \\
\hline \multirow{12}{*}{0} & \multirow{4}{*}{1} & 50 & 0 & 89.3 & 100 & 100 & 99.9 \\
\hline & & 100 & 0 & 80.1 & 100 & 100 & 99.4 \\
\hline & & 150 & 0 & 94.9 & 100 & 100 & 98.3 \\
\hline & & 200 & 0 & 96 & 100 & 100 & 97.5 \\
\hline & \multirow{4}{*}{3} & 50 & 0 & 93.3 & 100 & 100 & 99.9 \\
\hline & & 100 & 0 & 86.9 & 99.9 & 100 & 99.7 \\
\hline & & 150 & 0 & 87.7 & 100 & 100 & 99.9 \\
\hline & & 200 & 0 & 90.6 & 100 & 100 & 99.5 \\
\hline & \multirow{4}{*}{9} & 50 & 0 & 96.3 & 99.7 & 100 & 99.9 \\
\hline & & 100 & 0 & 92 & 99.9 & 100 & 99.8 \\
\hline & & 150 & 0 & 88.8 & 99.7 & 100 & 99.8 \\
\hline & & 200 & 0 & 88.4 & 99.9 & 100 & 99.7 \\
\hline \multirow{12}{*}{10} & \multirow{4}{*}{1} & 50 & 2 & 92.5 & 100 & 99.7 & 99.7 \\
\hline & & 100 & 2.7 & 82 & 99.9 & 98.6 & 99.1 \\
\hline & & 150 & 0.5 & 95.9 & 99.7 & 95.5 & 99.1 \\
\hline & & 200 & 0.7 & 97.5 & 98.8 & 92.3 & 98 \\
\hline & \multirow{4}{*}{3} & 50 & 0.3 & 93.8 & 99 & 99.8 & 99.9 \\
\hline & & 100 & 1.6 & 79.8 & 99.1 & 98.9 & 99.9 \\
\hline & & 150 & 1.4 & 87.9 & 98.7 & 97 & 99.3 \\
\hline & & 200 & 1.9 & 89.4 & 97.7 & 92 & 99.1 \\
\hline & \multirow{4}{*}{9} & 50 & 0.3 & 96.4 & 98.8 & 99.8 & 100 \\
\hline & & 100 & 0.6 & 82.3 & 97.6 & 99.1 & 99.9 \\
\hline & & 150 & 1.1 & 82.4 & 96.5 & 97.1 & 99.8 \\
\hline & & 200 & 2.1 & 81.6 & 92.1 & 92.4 & 99.8 \\
\hline \multirow{12}{*}{20} & \multirow{4}{*}{1} & 50 & 1.6 & 85.2 & 99.4 & 97.3 & 99.7 \\
\hline & & 100 & 5.3 & 80.6 & 99.9 & 95.4 & 98.8 \\
\hline & & 150 & 2.6 & 90.5 & 99.5 & 95 & 98.7 \\
\hline & & 200 & 0.9 & 96.6 & 99.8 & 94.1 & 96.9 \\
\hline & \multirow{4}{*}{3} & 50 & 0.6 & 89 & 94.2 & 96.4 & 99.8 \\
\hline & & 100 & 2.8 & 84.2 & 96.9 & 93.8 & 99.7 \\
\hline & & 150 & 3.9 & 82 & 97 & 93.5 & 99.4 \\
\hline & & 200 & 2.7 & 89.6 & 99.2 & 92.9 & 98.5 \\
\hline & \multirow{4}{*}{9} & 50 & 0.7 & 91.6 & 88.7 & 95.4 & 100 \\
\hline & & 100 & 0.9 & 86.3 & 87.3 & 93.9 & 100 \\
\hline & & 150 & 1.8 & 88.2 & 86.1 & 92.9 & 99.8 \\
\hline & & 200 & 2.6 & 86.7 & 91.8 & 92.2 & 99.3 \\
\hline \multirow{12}{*}{30} & \multirow{4}{*}{1} & 50 & 4.6 & 51.6 & 99.5 & 97.3 & 99.8 \\
\hline & & 100 & 9.3 & 76.9 & 99.9 & 96.1 & 99.6 \\
\hline & & 150 & 3.7 & 88.8 & 99.7 & 95.4 & 97.9 \\
\hline & & 200 & 0.8 & 94.9 & 100 & 94.9 & 97.8 \\
\hline & \multirow{4}{*}{3} & 50 & 2.7 & 80.5 & 97 & 96.2 & 99.9 \\
\hline & & 100 & 1.7 & 87.8 & 96.1 & 94.4 & 99.9 \\
\hline & & 150 & 5.1 & 82.2 & 98.7 & 93.1 & 99.4 \\
\hline & & 200 & 1.1 & 95.6 & 99.6 & 90.8 & 99.1 \\
\hline & \multirow{4}{*}{9} & 50 & 1.2 & 87.4 & 90.9 & 95.7 & 100 \\
\hline & & 100 & 1.6 & 91.6 & 89.9 & 93.5 & 99.9 \\
\hline & & 150 & 1.8 & 87.3 & 94.9 & 94.3 & 99.9 \\
\hline & & 200 & 2.8 & 89.4 & 98.9 & 90.3 & 99.5 \\
\hline
\end{tabular}

change in $\beta_{4}$. Similarly, after deleting the 14th, 21st, 22nd, 24 th, and 25 th observations from the data set, $\beta_{4}$ shows the maximum absolute changes as $381.0066 \%$, $92.6017 \%$, $694.1877 \%, 183.8186 \%$, and $260.3238 \%$, respectively.

Table 5 and Figure 4 show that the influence and curvature diagnostics for LNRM for the example data are based on $G C D_{i}$, One-Step NR diagnostics, and curvature diagnostics. $\mathrm{GCD}_{i}$ detects 1 st and 6 th as influential observation. One-Step NR diagnostics based on $x_{1}$ detect 1st, 2nd, 3rd, 8th, 11th, 22nd, 24th, and 25th as influential observations, while based on other three variables, NR detects observations 9th, 13th, 14th, 20th, 21st, and 23rd as potential influential observations. The case weight perturbation shows 1st, 13th, 14th, 21st, 22nd, and 24th observations are the most distinguished as compared to other observations. 
Table 3: Percentage of influential diagnostics at $p=4$.

\begin{tabular}{|c|c|c|c|c|c|c|c|}
\hline Censoring (\%) & $\sigma$ & $n$ & GCD & $\mathrm{NR}_{x_{2}}$ & $\mathrm{CV}_{\text {case weight }}$ & $C V_{\text {response }}$ & $\mathrm{CV}_{\text {covariate }}$ \\
\hline \multirow{12}{*}{0} & \multirow{4}{*}{1} & 50 & 0 & 62.8 & 100 & 100 & 99.6 \\
\hline & & 100 & 0 & 90.6 & 100 & 100 & 99.7 \\
\hline & & 150 & 0 & 96.1 & 100 & 100 & 99.2 \\
\hline & & 200 & 0 & 95.5 & 100 & 100 & 99 \\
\hline & \multirow{4}{*}{3} & 50 & 0 & 84.3 & 99.8 & 100 & 99.9 \\
\hline & & 100 & 0 & 83.8 & 99.9 & 100 & 99.6 \\
\hline & & 150 & 0 & 87.8 & 100 & 100 & 99.4 \\
\hline & & 200 & 0 & 93.8 & 100 & 100 & 99.4 \\
\hline & \multirow{4}{*}{9} & 50 & 0 & 94.5 & 99.9 & 100 & 100 \\
\hline & & 100 & 0 & 88.3 & 99.8 & 100 & 100 \\
\hline & & 150 & 0 & 88.5 & 99.8 & 100 & 100 \\
\hline & & 200 & 0 & 89.6 & 99.7 & 100 & 99.6 \\
\hline \multirow{12}{*}{10} & \multirow{4}{*}{1} & 50 & 6.6 & 78.3 & 99.8 & 99.3 & 99.7 \\
\hline & & 100 & 5.1 & 81.1 & 99.9 & 99.3 & 99.8 \\
\hline & & 150 & 1.8 & 92.6 & 99.9 & 99.3 & 98.9 \\
\hline & & 200 & 1.6 & 96.5 & 100 & 99.1 & 99.1 \\
\hline & \multirow{4}{*}{3} & 50 & 0.9 & 86.7 & 99.4 & 99.4 & 99.8 \\
\hline & & 100 & 9.3 & 72.2 & 99.9 & 99.1 & 99.9 \\
\hline & & 150 & 2.4 & 88.7 & 99.9 & 98.8 & 99.6 \\
\hline & & 200 & 4 & 90.9 & 99.7 & 98.9 & 99.4 \\
\hline & \multirow{4}{*}{9} & 50 & 0.4 & 92.1 & 99 & 99.7 & 99.9 \\
\hline & & 100 & 3.2 & 76 & 98.9 & 99.5 & 100 \\
\hline & & 150 & 2.8 & 78.9 & 99.3 & 98.7 & 99.8 \\
\hline & & 200 & 4 & 80.5 & 99.1 & 98.5 & 100 \\
\hline \multirow{12}{*}{20} & \multirow{4}{*}{1} & 50 & 34.4 & 39.3 & 99.9 & 99.7 & 99.9 \\
\hline & & 100 & 18.1 & 79.3 & 99.8 & 99.1 & 99.7 \\
\hline & & 150 & 6.6 & 90.9 & 99.8 & 98 & 99.3 \\
\hline & & 200 & 2.3 & 94.9 & 99.9 & 98.1 & 98.9 \\
\hline & \multirow{4}{*}{3} & 50 & 8.8 & 66.9 & 99.2 & 99.6 & 100 \\
\hline & & 100 & 12.8 & 74.2 & 99.5 & 98.9 & 99.9 \\
\hline & & 150 & 12.1 & 79.7 & 99.7 & 98.9 & 99.9 \\
\hline & & 200 & 3.8 & 92.2 & 99.9 & 97.9 & 99.3 \\
\hline & \multirow{4}{*}{9} & 50 & 3.1 & 81.5 & 98.6 & 99.5 & 100 \\
\hline & & 100 & 3.2 & 82 & 98.4 & 99.4 & 100 \\
\hline & & 150 & 5.2 & 82.8 & 98.1 & 97.7 & 99.9 \\
\hline & & 200 & 4.7 & 85.6 & 99 & 97.6 & 99.7 \\
\hline \multirow{12}{*}{30} & \multirow{4}{*}{1} & 50 & 46.8 & 34.6 & 100 & 99.3 & 99.8 \\
\hline & & 100 & 34.1 & 62.2 & 100 & 98.9 & 99.7 \\
\hline & & 150 & 6.8 & 88.4 & 99.9 & 96.9 & 99.2 \\
\hline & & 200 & 0.8 & 94.5 & 100 & 96.7 & 98.6 \\
\hline & \multirow{4}{*}{3} & 50 & 14.6 & 56.6 & 99.2 & 99 & 100 \\
\hline & & 100 & 13 & 78.8 & 99 & 98.7 & 100 \\
\hline & & 150 & 11.9 & 78.7 & 99.7 & 97.8 & 100 \\
\hline & & 200 & 1.6 & 93.6 & 100 & 97.3 & 99.3 \\
\hline & \multirow{4}{*}{9} & 50 & 4.6 & 82.9 & 99 & 99.3 & 100 \\
\hline & & 100 & 4.8 & 87.2 & 98.5 & 98 & 100 \\
\hline & & 150 & 6.2 & 85.2 & 97.2 & 96.5 & 100 \\
\hline & & 200 & 2.3 & 91 & 98.9 & 96.8 & 99.7 \\
\hline
\end{tabular}

Next, the influence of perturbations on the observed survival times will be examined. For response curvature, the 14 th and 21st observations are distinguished from the other observations.
The perturbation of vectors for covariates, $x_{1 i}, x_{2 i}, x_{3 i}$, and $x_{4 i}$, is investigated here. For perturbation of covariates, observations $2 \mathrm{nd}, 8$ th, 11 th, and 20 th are observations with high influence. 

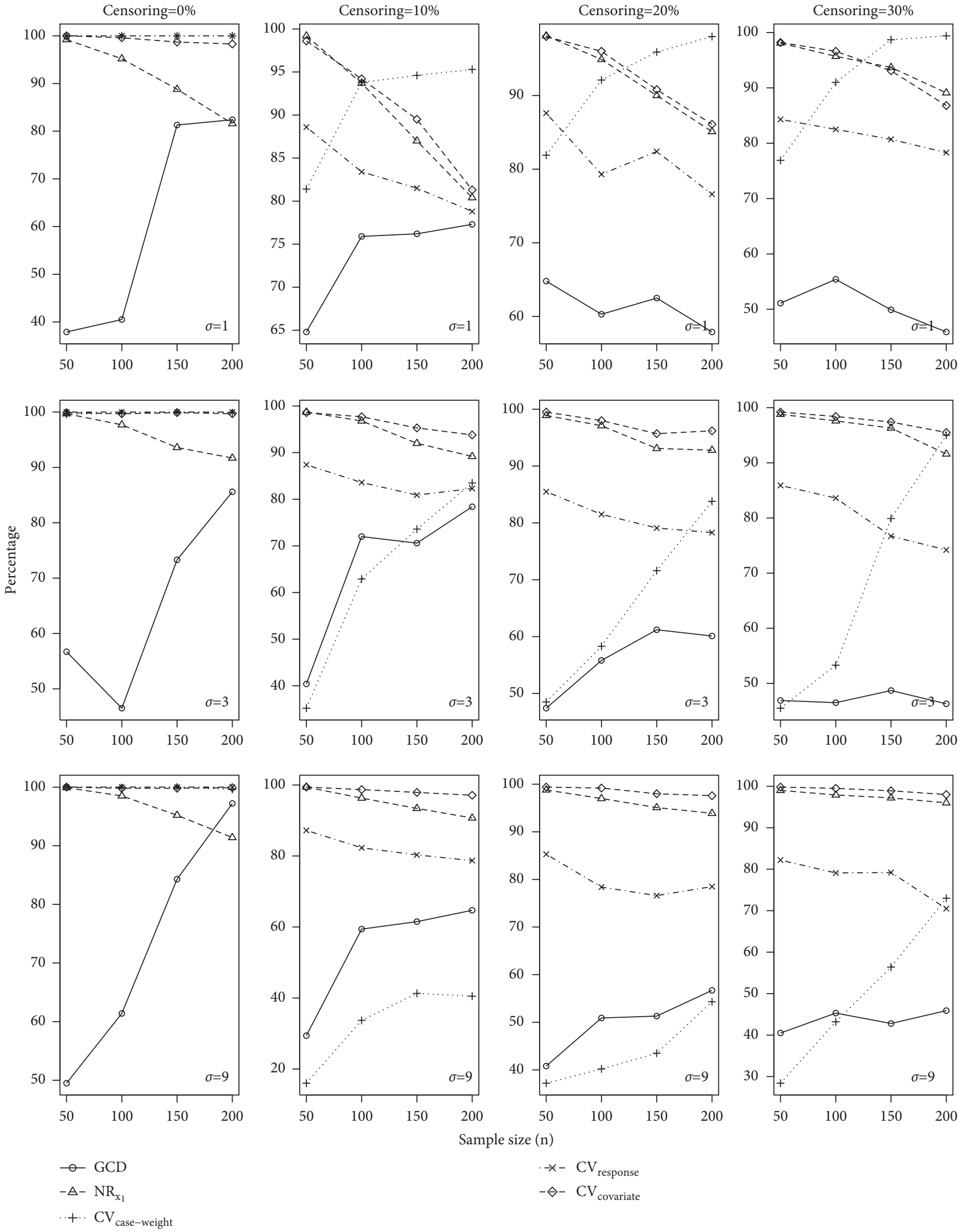

$$
\begin{aligned}
& -x-C_{\text {response }} \\
& -\diamond-C_{\text {covariate }}
\end{aligned}
$$

Figure 1: Percentage of influential diagnostics at $p=1$. 

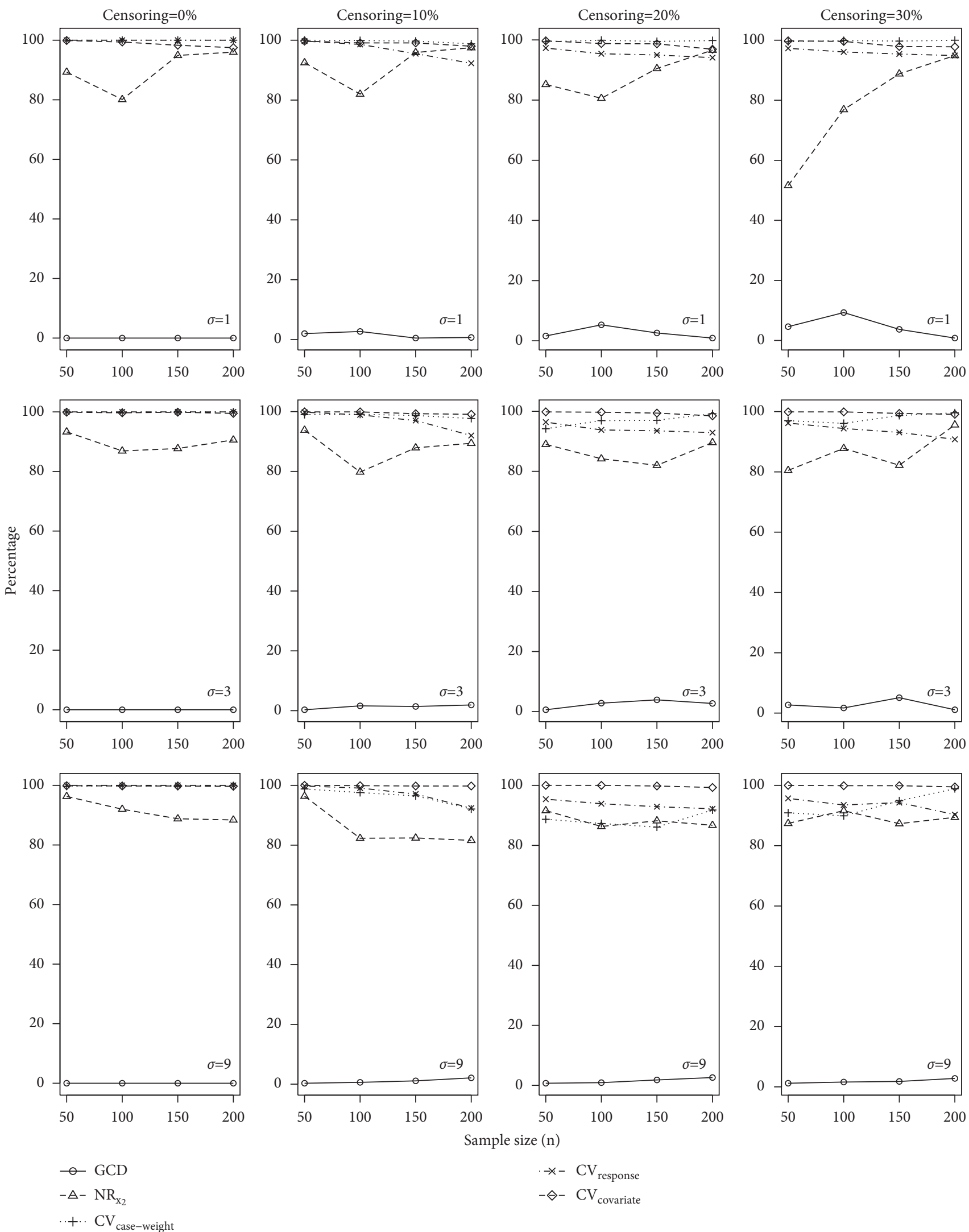

$-\diamond-\mathrm{CV}_{\text {covariate }}$

Figure 2: Percentage of influential diagnostics at $p=2$. 

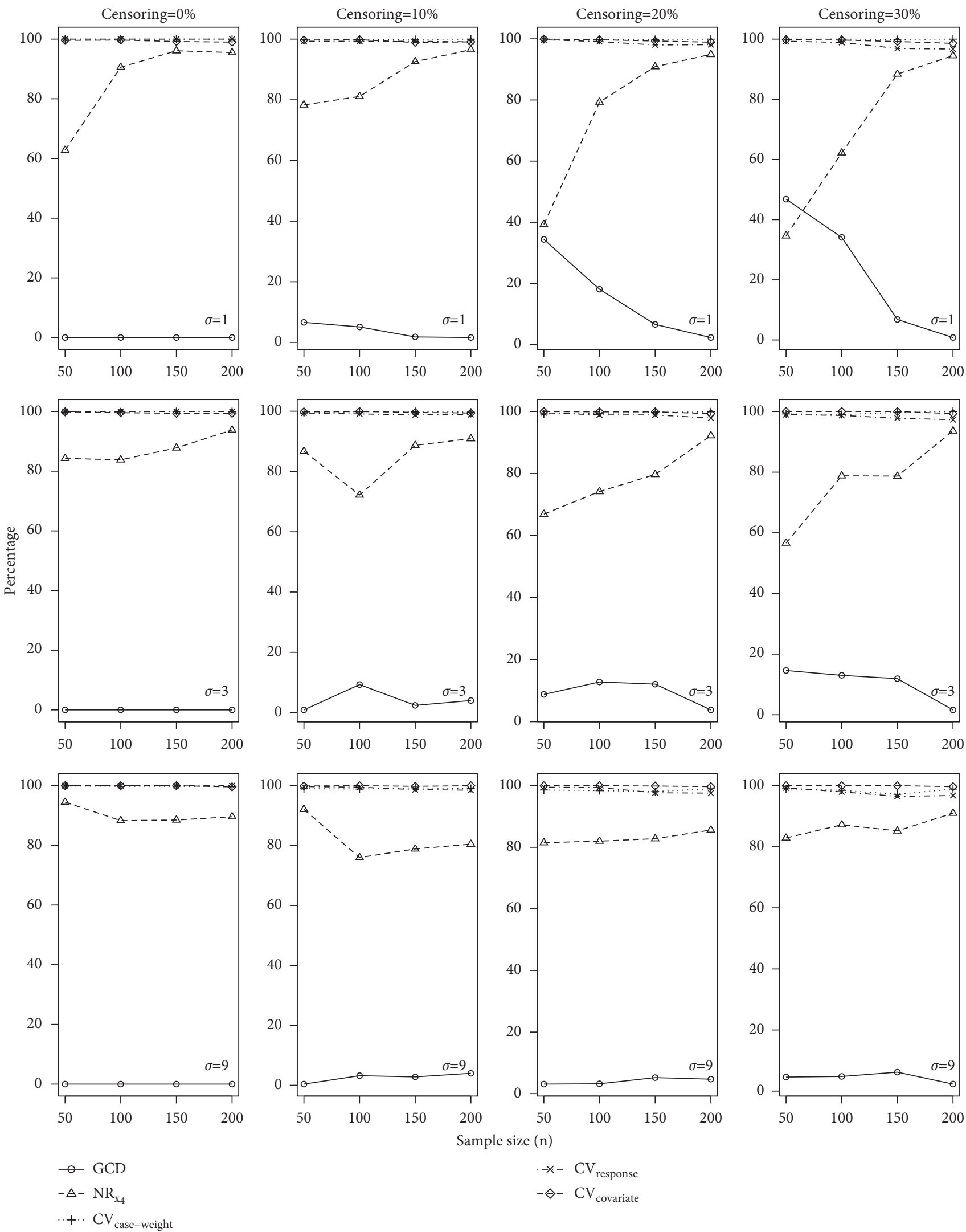

$$
\begin{aligned}
& \cdot-\rtimes-\mathrm{CV}_{\text {response }} \\
& -\diamond-\mathrm{CV}_{\text {covariate }}
\end{aligned}
$$

FIgURE 3: Percentage of influential diagnostics at $p=4$. 


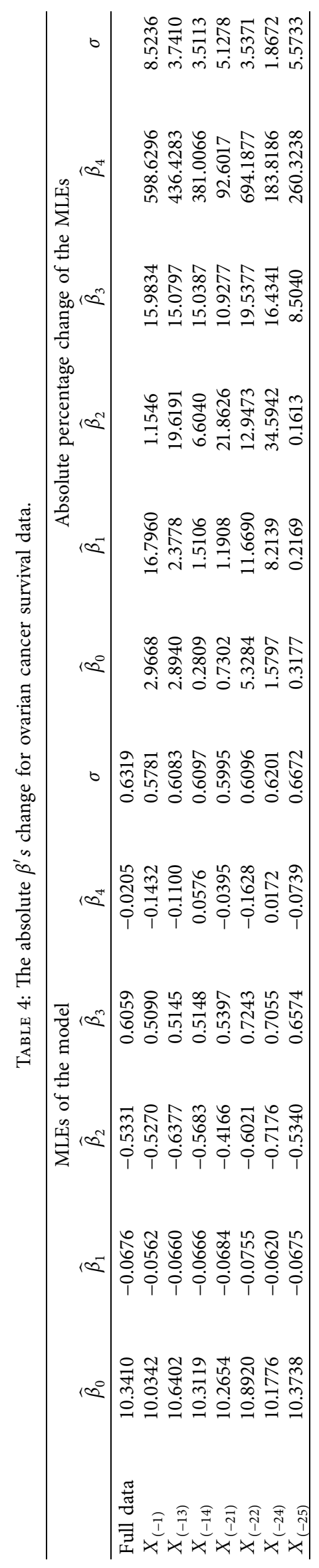




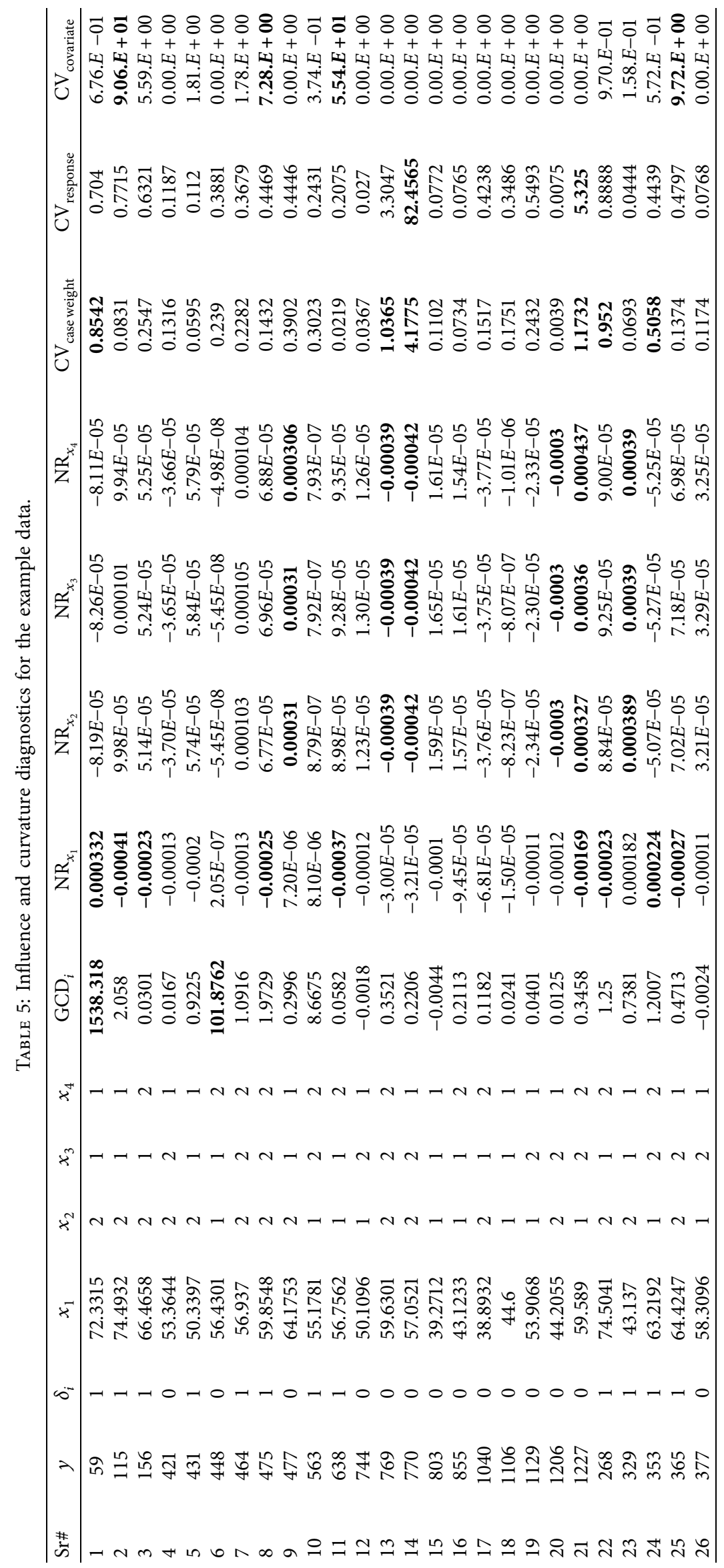




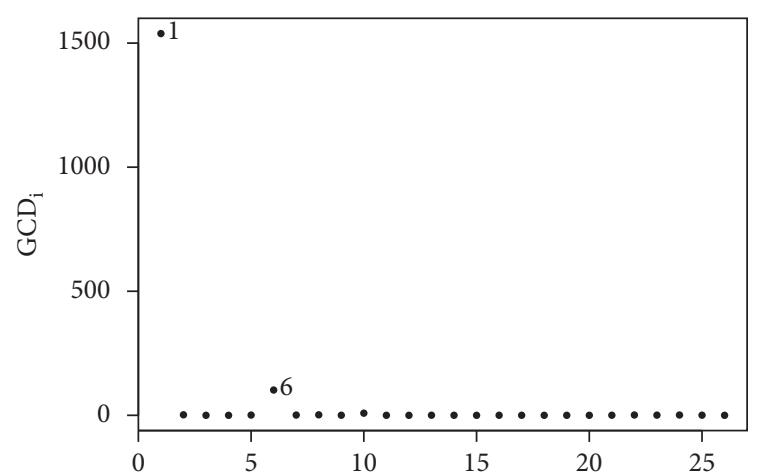

(a)

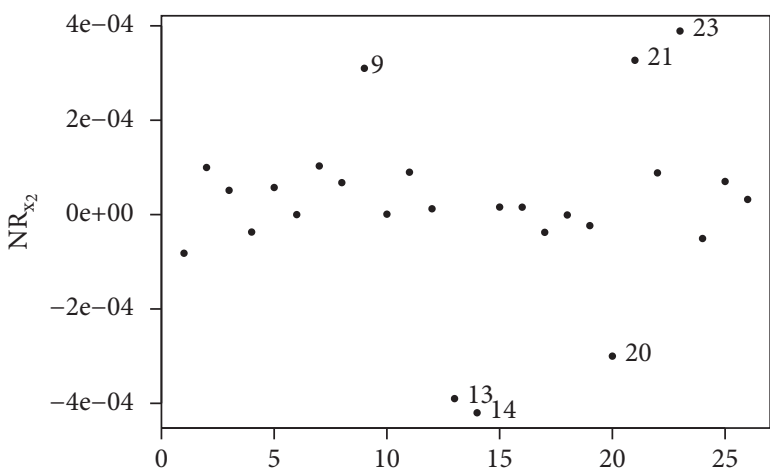

(c)

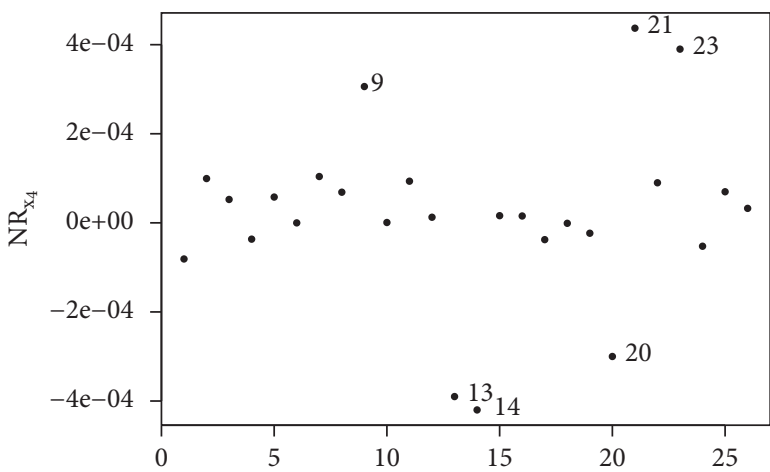

(e)

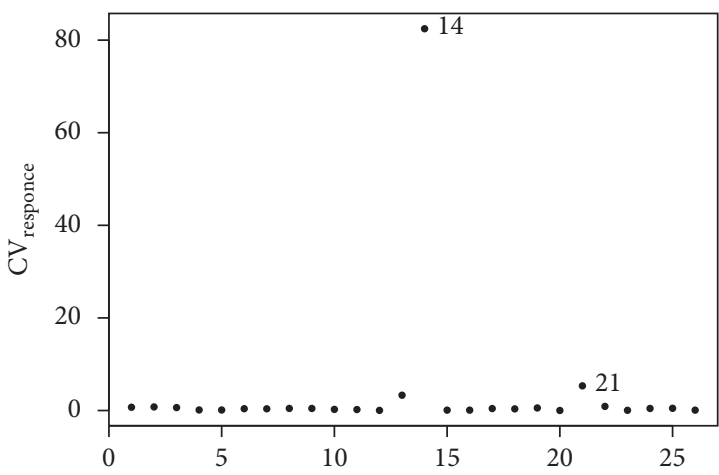

(g)

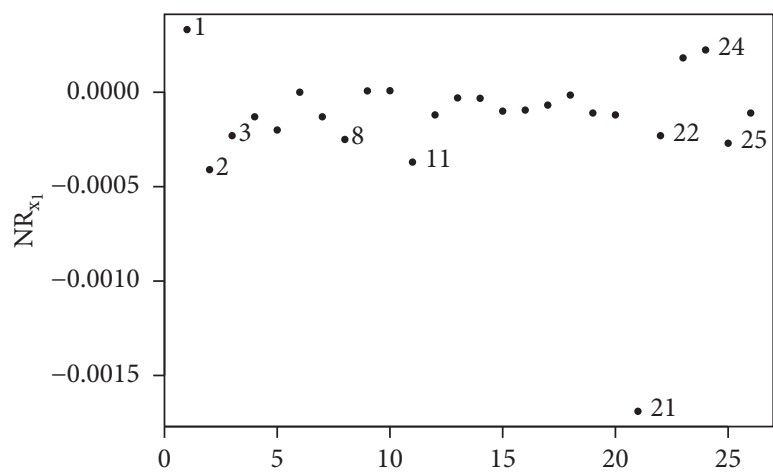

(b)

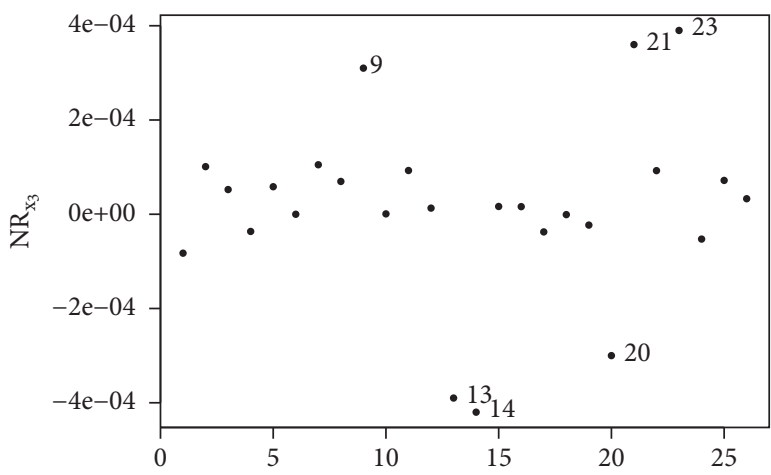

(d)

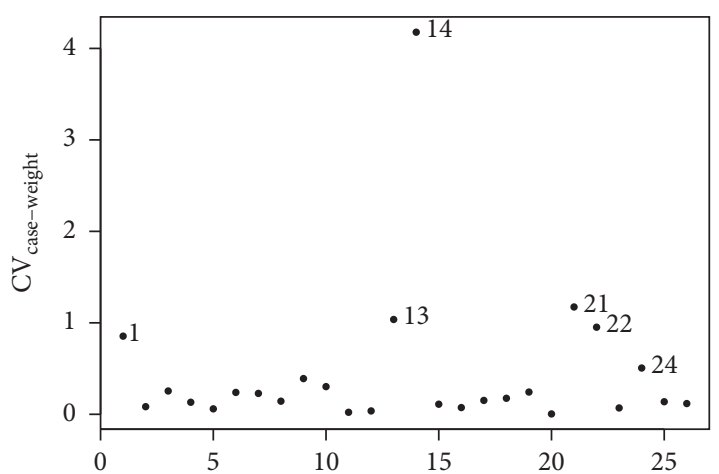

(f)

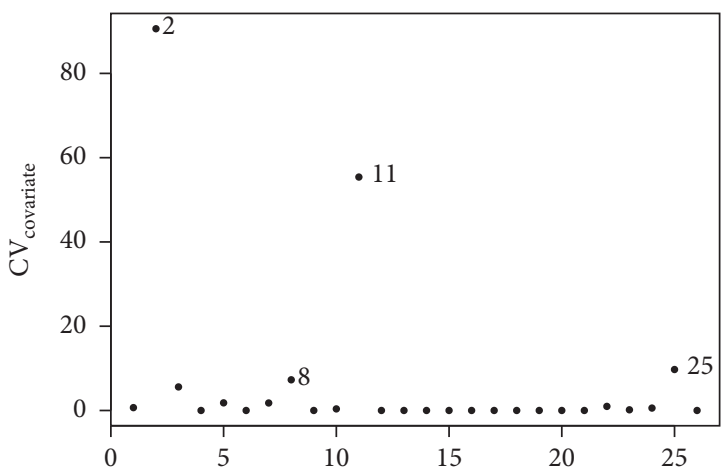

(h)

Figure 4: Influence and curvature diagnostics for the example data. 


\section{Concluding Remarks}

This paper developed new diagnostic approaches for the LNRM with censored data to identify the influential observations by using the LI technique. The curvatures were obtained as a measure of local influence under the perturbation scheme of case weight $\left(\mathrm{CV}_{\text {Case weight }}\right)$, response $\left(\mathrm{CV}_{\text {response }}\right)$, and explanatory variables $\left(\mathrm{CV}_{\text {covariate }}\right)$. We accomplish the global influence methods based on $\mathrm{GCD}_{i}$ and One-Step NR method. $\mathrm{CV}_{\text {case weight }}$ is observed superior in simulation when there is $0 \%$ censoring in the data. While increasing the level of censoring, the performance of $\mathrm{CV}_{\text {covariates }}$ was better than the others.

\section{Appendix}

\section{A. First-Order Partial Derivatives $(q(\widehat{\boldsymbol{\theta}}))$}

Here, we derive the necessary computation formulas to obtain the first-order partial derivatives of the log-likelihood function. After a few algebraic manipulations,

$$
\begin{aligned}
\frac{\partial l}{\partial \beta} & =\sum\left[\frac{X^{\prime}}{\sigma}\left\{\delta_{i}\left(\frac{y-X^{\prime} \beta}{\sigma}\right)+\frac{1}{\left(\left(y-X^{\prime} \beta\right) / \sigma\right)}-\frac{\delta_{i}}{\left(\left(y-X^{\prime} \beta\right) / \sigma\right)}\right\}\right], \\
\frac{\partial l}{\partial \sigma} & =\sum\left[\frac{1}{\sigma}+\frac{\delta_{i} \omega_{i}^{2}}{\sigma}-2 \frac{\delta_{i}}{\sigma}\right], \\
q(\hat{\theta}) & =\left\{\begin{array}{l}
\sum\left[\frac{X^{\prime}}{\sigma}\left(\delta_{i} \omega_{i}+\frac{1}{\omega_{i}}-\frac{\delta_{i}}{\omega_{i}}\right)\right], \\
\sum\left[\frac{1}{\sigma}+\frac{\delta_{i} \omega_{i}^{2}}{\sigma}-\frac{2 \delta_{i}}{\sigma}\right],
\end{array}\right.
\end{aligned}
$$

where $w_{i}=\left(\left(y_{i}-x_{i}^{T} \beta\right) / \sigma\right)$.

\section{B. Second-Order Partial Derivatives $(\ddot{L}(\boldsymbol{\theta}))$}

Here, we derive the necessary formulas to obtain the secondorder partial derivatives of the log-likelihood function. After some algebraic manipulations, we obtain

$$
\begin{aligned}
L_{\beta \beta} & =\frac{\partial^{2} l}{\partial \beta^{2}}=\sum\left[\frac{X^{\prime 2}}{\sigma^{2}}\left\{-\delta_{i}+\left(\frac{y-X^{\prime} \beta}{\sigma}\right)^{-2}-\delta_{i}\left(\frac{y-X^{\prime} \beta}{\sigma}\right)^{-2}\right\}\right], \quad k, j=1, \ldots, p, \\
L_{\beta \sigma}=\frac{\partial^{2} l}{\partial \beta \partial \sigma}= & \sum\left[-\delta_{i} X^{\prime}\left(\frac{y-X^{\prime} \beta}{\sigma}\right)\left(1+\frac{1}{\sigma^{2}}\right)\right], \\
L_{\sigma \sigma} & =\frac{\partial^{2} l}{\partial \sigma^{2}}=\sum\left[\frac{1}{\sigma^{2}}\left(2 \delta_{i}-3 \delta_{i} \omega_{i}^{2}-1\right)\right], \\
I(\theta) & =-\left[\begin{array}{c}
\left.\sum\left[\frac{X^{\prime}}{\sigma^{2}}\left(-\delta_{i}+\omega_{i}^{-2}-\delta_{i} \omega_{i}^{-2}\right)\right] \sum\left[-\frac{\delta_{i} X^{\prime}}{\sigma^{2}}\left(\frac{1+\omega_{i}^{2}}{\omega_{i}^{2}}\right)\right]\right]^{-1} \\
\sum\left[-\frac{\delta_{i} X^{\prime}}{\sigma^{2}}\left(\frac{1+\omega_{i}^{2}}{\omega_{i}^{2}}\right)\right] \sum\left[\frac{1}{\sigma^{2}}\left(2 \delta_{i}-3 \delta_{i} \omega_{i}^{2}-1\right)\right]
\end{array}\right]
\end{aligned}
$$

\section{Data Availability}

The data are available from the first author upon request.

\section{Conflicts of Interest}

The authors declare that they have no conflicts of interest.

\section{References}

[1] C. B. Williams, "An analysis of four years captures of insects in a light trap. Part 1I.1 the effect of weather conditions on insect activity; and the estimation and forecasting of changes in the insect population," Transactions of the Royal Entomological Society of London, vol. 90, no. 8, pp. 227-306, 1940.

[2] R. Shanker, "Lognormal distribution and its applications in biological and medical sciences," in Proceedings of the 4th International Conference and Exhibition on Biometrics and Biostatistics, San Antonio, TX, USA, November 2015.
[3] Z. Jin, D. Y. Lin, L. J. Wei, and Z. Ying, "Rank-based inference for the accelerated failure time model," Biometrika, vol. 90, no. 2, pp. 341-353, 2003.

[4] Z. Ma and E. J. Bechinski, "Accelerated failure time (AFT) modeling for the development and survival of Russian wheat aphid, Diuraphis noxia (Mordvilko)," Population Ecology, vol. 51, no. 4, pp. 543-548, 2009.

[5] D. R. Cox and D. Oakes, Analysis of Survival Data, Vol. 21, CRC Press, , Boca Raton, FL, USA, 1984.

[6] J. D. Kalbfleisch and R. L. Prentice, The Statistical Analysis of Failure Time Data, Vol. 360, John Wiley \& Sons, , Hoboken, NJ, USA, 2011.

[7] J. Aitchison and J. Brown, The Lognormal Distribution, Cambridge University Press, Cambridge, UK, 1963.

[8] E. Limpert, W. A. Stahel, and M. Abbt, "Log-normal distributions across the sciences: keys and clues," BioScience, vol. 51, no. 5, pp. 341-352, 2001.

[9] M. Mitzenmacher, "A brief history of generative models for power law and lognormal distributions," Internet Mathematics, vol. 1, no. 2, pp. 226-251, 2004. 
[10] P. Tai, J. Tonita, E. Yu, and D. Skarsgard, “Twenty-year followup study of long-term survival of limited-stage small-cell lung cancer and overview of prognostic and treatment factors," International Journal of Radiation Oncology, Biology, Physics, vol. 56, no. 3, pp. 626-633, 2003.

[11] T. Kuroishi, S. Tominaga, T. Morimoto et al., "Tumor growth rate and prognosis of breast cancer mainly detected by mass screening," Japanese Journal of Cancer Research, vol. 81, no. 5, pp. 454-462, 1990.

[12] H. Weedon-Fekjær, B. H. Lindqvist, L. J. Vatten, O. O. Aalen, and S. Tretli, "Breast cancer tumor growth estimated through mammography screening data," Breast Cancer Research, vol. 10, no. 3, 2008.

[13] W. D. Stein, W. D. Figg, W. Dahut et al., "Tumor growth rates derived from data for patients in a clinical trial correlate strongly with patient survival: a novel strategy for evaluation of clinical trial data," The Oncologist, vol. 13, no. 10, pp. 1046-1054, 2008.

[14] J. Wilkerson, K. Abdallah, C. Hugh-Jones et al., "Estimation of tumour regression and growth rates during treatment in patients with advanced prostate cancer: a retrospective analysis," The Lancet Oncology, vol. 18, no. 1, pp. 143-154, 2017.

[15] N. M. Linton, T. Kobayashi, Y. Yang et al., "Incubation period and other epidemiological characteristics of 2019 novel coronavirus infections with right truncation: a statistical analysis of publicly available case data," Journal of Clinical Medicine, vol. 9, no. 22, p. 538, 2020.

[16] J. Odhiambo, P. Weke, and J. Wendo, "Modeling of returns of nairobi securities exchange 20 share index using log-normal distribution," Research Journal of Finance and Accounting, vol. 11, no. 8, 2020.

[17] A. L. Sweet, "On the hazard rate of the lognormal distribution," IEEE Transactions on Reliability, vol. 39, no. 3, pp. 325-328, 1990.

[18] S. Chatterjee and A. S. Hadi, "Impact of simultaneous omission of a variable and an observation on a linear regression equation," Computational Statistics \& Data Analysis, vol. 6, no. 2, pp. 129-144, 1988.

[19] R. D. Cook, "Detection of influential observation in linear regression," Technometrics, vol. 19, no. 1, pp. 15-18, 1977.

[20] H. Zhu, S. Y. Lee, B. C. Wei, and J. Zhou, "Case-deletion measures for models with incomplete data," Biometrika, vol. 88 , no. 3, pp. 727-737, 2001.

[21] H. Zhu, J. G. Ibrahim, S. Lee, and H. Zhang, "Perturbation selection and influence measures in local influence analysis," Annals of Statistics, vol. 35, no. 6, pp. 2565-2588, 2007.

[22] H. Zhu, J. G. Ibrahim, and X. Shi, "Diagnostic measures for generalized linear models with missing covariates," Scandinavian Journal of Statistics, vol. 36, no. 4, pp. 686-712, 2009.

[23] M. B. Massuia, C. R. B. Cabral, L. A. Matos, and V. H. Lachos, "Influence diagnostics for Student-tcensored linear regression models," Statistics, vol. 49, no. 5, pp. 1074-1094, 2015.

[24] R. D. Cook, "Assessment of local influence," Journal of the Royal Statistical Society: Series B, vol. 48, no. 2, pp. 133-155, 1986.

[25] L. A. Escobar and W. Q. Meeker Jr, "Assessing influence in regression analysis with censored data," Biometrics, vol. 48, no. 2, pp. 507-528, 1992.

[26] L. A. Weissfeld and H. Schneider, "Influence diagnostics for the Weibull model fit to censored data," Statistics \& Probability Letters, vol. 9, no. 1, pp. 67-73, 1990.

[27] L. A. Weissfeld and H. Schneider, "Influence diagnostics for the normal linear model with censored data," Australian Journal of Statistics, vol. 32, no. 1, pp. 11-20, 1990.
[28] V. Leiva, M. Barros, G. A. Paula, and M. Galea, "Influence diagnostics in log-Birnbaum-Saunders regression models with censored data," Computational Statistics \& Data Analysis, vol. 51, no. 12, pp. 5694-5707, 2007.

[29] E. M. Ortega, V. G. Cancho, and H. Bolfarine, "Influence diagnostics in exponentiated-Weibull regression models with censored data," Statistics and Operations Research Transactions, vol. 30, pp. 171-192, 2006.

[30] S. Liu, "On local influence for elliptical linear models," Statistical Papers, vol. 41, no. 2, pp. 211-224, 2000.

[31] E. M. Ortega, H. Bolfarine, and G. A. Paula, "Influence diagnostics in generalized log-gamma regression models," Computational Statistics and Data Analysis, vol. 42, no. 1-2, pp. 165-186, 2003.

[32] M. K. Venezuela, M. C. Sandoval, and D. A. Botter, "Local influence in estimating equations," Computational Statistics \& Data Analysis, vol. 55, no. 4, pp. 1867-1883, 2011.

[33] V. H. Lachos, F. Vilca, and M. Galea, "Influence diagnostics for the Grubbs's model," Statistical Papers, vol. 48, no. 3, pp. 419-436, 2007.

[34] G. A. Paula, M. Medeiros, and F. E. Vilca-Labra, "Influence diagnostics for linear models with first-order autoregressive elliptical errors," Statistics \& Probability Letters, vol. 79, no. 3, pp. 339-346, 2009.

[35] C. M. Russo, G. A. Paula, and R. Aoki, "Influence diagnostics in nonlinear mixed-effects elliptical models," Computational Statistics \& Data Analysis, vol. 53, no. 12, pp. 4143-4156, 2009.

[36] L. H. Vanegas, L. M. Rondón, and G. M. Cordeiro, "Diagnostic tools in generalized Weibull linear regression models," Journal of Statistical Computation and Simulation, vol. 83, no. 12, pp. 2315-2338, 2013.

[37] J. Khaleeq, M. Amanullah, A. T. Abdulrahman, E. H. Hafez, and M. M. Abd El-Raouf, "Influence diagnostics in LogLogistic regression model with censored data," Alexandria Engineering Journal, vol. 61, no. 3, 2021.

[38] R. D. Cook and S. Weisberg, Residuals and Influence in Regression, Chapman and Hall, New York, NY, USA, 1982.

[39] D. A. Belsley, E. Kuh, and R. E. Welsch, Regression Diagnostics: Identifying Influential Data and Sources of Collinearity, John Wiley \& Sons, Hoboken, NJ, USA, 2005.

[40] H. J. Edmonson, J. Su, and J. E. Krook, "Treatment of ovarian cancer in elderly women: Mayo clinic-north central cancer treatment group studies," Cancer, vol. 71, no. S2, pp. 615-617, 1993. 